\title{
Stent Retrievers in Acute Ischemic Stroke: Complications and Failures during the Perioperative Period
}

\author{
G. Gascou, K. Lobotesis, P. Machi, I. Maldonado, J.F. Vendrell, C. Riquelme, O. Eker, G. Mercier, I. Mourand,
}

C. Arquizan, A. Bonafé, and V. Costalat

\begin{abstract}
BACKGROUND AND PURPOSE: Stent retriever-assisted thrombectomy promotes high recanalization rates in acute ischemic stroke. Nevertheless, complications and failures occur in more than $10 \%$ of procedures; hence, there is a need for further investigation.

MATERIALS AND METHODS: A total of 144 patients with ischemic stroke presenting with large-vessel occlusion were prospectively included. Patients were treated with stent retriever-assisted thrombectomy \pm IV fibrinolysis. Baseline clinical and imaging characteristics were incorporated in univariate and multivariate analyses. Predictors of recanalization failure ( $\mathrm{TICl} 0,1,2 \mathrm{a})$, and of embolic and hemorrhagic complications were reported. The relationship between complication occurrence and periprocedural mortality rate was studied.

RESULTS: Median age was 69.5 years, and median NIHSS score was 18 at presentation. Fifty patients (34.7\%) received stand-alone thrombectomy, and 94 (65.3\%) received combined therapy. The procedural failure rate was $13.9 \%$. Embolic complications were recorded in $12.5 \%$ and symptomatic intracranial hemorrhage in $7.6 \%$. The overall rate of failure, complications, and/or death was $39.6 \%$. The perioperative mortality rate was $18.4 \%$ in the overall cohort but was higher in cases of failure (45\%; $P=.003)$, embolic complications ( $38.9 \%$; $P=.0176)$, symptomatic intracranial hemorrhages (45.5\%; $P=.0236)$, and intracranial stenosis $(50 \% ; P=.0176)$. Concomitant fibrinolytic therapy did not influence the rate of recanalization or embolic complication, or the intracranial hemorrhage rate. Age was the only significant predictive factor of intracranial hemorrhage $(P=.043)$.
\end{abstract}

CONCLUSIONS: The rate of perioperative mortality was significantly increased in cases of embolic and hemorrhagic complications, as well as in cases of failure and underlying intracranial stenoses. Adjunctive fibrinolytic therapy did not improve the recanalization rate or collateral embolic complication rate. The rate of symptomatic intracranial hemorrhage was not increased in cases of combined treatment.

ABBREVIATIONS: BA = basilar artery; BGC = balloon-guided catheter; ECASS II = European Co-operative Acute Stroke Study-II; HI = hemorrhagic infarct; ICH = intracranial hemorrhage; $\mathrm{PH}=$ parenchymatous hemorrhage

V fibrinolysis within 4.5 hours after stroke onset is the reference therapy for acute ischemic stroke in the Western world. Many predictors of success or failure have been reported in relationship

Received March 9, 2013; accepted after revision July 24.

From CHU Montpellier, Neuroradiology (G.G., P.M., I.M., J.F.V., C.R., O.E., A.B., V.C.) and Neurology (C.A., I.M.), Montpellier, France; Imaging Department (K.L.), Imperial College Healthcare NHS Trust, Charing Cross Hospital, London, United Kingdom; CHU Montpellier, Department of Medical Statistics (G.M.), Arnaud de Villeneuve Hospital, University of Montpellier, Montpellier, France.

All authors have made substantial contributions to all of the following: the conception and design of the study (G.G., V.C., A.B., P.M.), acquisition of data (G.G., V.C., P.M., C.R., O.E., A.B., I.M., C.A., J.F.V.), analysis and interpretation of data (G.M., G.G., V.C., K.L., A.B.), and drafting the article or revising it critically for important intellectual content (G.G., V.C., K.L., A.B.).

Please address correspondence to Vincent Costalat, MD, PhD, CHU Montpellier Neuroradiology, Av Augustin Fliche, Montpellier, France; e-mail: v-costalat@ chu-montpellier.fr

三 Indicates article with supplemental on-line table.

http://dx.doi.org/10.3174/ajnr.A3746 to this treatment. ${ }^{1-4}$ Proximal intracranial artery occlusions, ${ }^{5,6}$ cardiovascular risk factors, and high NIHSS score have all been reported to be associated with failed recanalization after IV fibrinolysis. ${ }^{3,6-8}$ Symptomatic risk for intracranial hemorrhage (ICH) increases gradually with increasing infarct size and patient age. ${ }^{9,10}$ Mechanical thrombectomy is an adjuvant or alternative therapy for acute ischemic stroke when IV fibrinolysis is contraindicated or has failed. Recent studies ${ }^{11-17}$ have shown that mechanical thrombectomy by use of a stent retriever is successful in achieving a high rate of arterial recanalization, with a low complication rate. This technique is promising; however, adverse embolic and hemorrhagic events have been reported in roughly $5 \%-10 \%{ }^{12,14}$ of procedures, with a failure rate ranging from $9 \%-33 \% .{ }^{11,17,18}$ With this in mind, we reviewed our prospective data base of 144 consecutive patients to identify predictive factors of failure, complications, and periprocedural mortality. 


\section{MATERIALS AND METHODS}

\section{Patient Selection}

Ethical approval for this study was obtained by the local ethics committee in our institution after review of the stroke protocol. Patients with acute stroke were examined at admission by a senior stroke neurologist who assessed the severity of the neurologic deficit by using the NIHSS. An NIHSS score equal or superior to 8 was required for mechanical thrombectomy to be considered. Patients underwent comprehensive imaging in the acute phase, with MR imaging to delineate the extent of the ischemic lesion. In cases of MR imaging contraindication, CT, CTA, and CTP were carried out.

In patients with anterior circulation strokes, the ASPECT score was calculated on DWI MR imaging or CT to assess the extent of the ischemic core. Only patients with an ASPECT score of $\geq 5$ were included, according to our institutional protocol ${ }^{12}$ and the SAMURAI (Stroke Acute Management with Urgent Riskfactor Assessment and Improvement) study. ${ }^{19}$

In accordance with our institutional stroke protocol, patients presenting within 4.5 hours from symptom onset received a combined therapy associating IV fibrinolysis ( $\mathrm{rtPA} 0.9 \mathrm{mg} / \mathrm{kg}$ ) and mechanical thrombectomy. Patient presenting between 4.5 and 6 hours with anterior circulation strokes or presenting with IV fibrinolysis contraindication were treated with stand-alone thrombectomy. For patients with posterior circulation strokes, a combined approach was performed up to 24 hours after symptom onset, depending on the extent of the ischemic lesions. Extensive brain stem lesions (eg, bilateral complete involvement of the pons, mesencephalon, or diencephalic structures) were the main exclusion criteria in this location.

\section{MR Imaging Protocol}

MR imaging was performed by use of a $1.5 \mathrm{~T}$ magnet $(33 \mathrm{mT} / \mathrm{m}$ hypergradients; Intera, release 10; Philips Healthcare, Best, the Netherlands) with a phased-array head coil. First, a T2 gradientecho was performed to screen for ICH. Then, DWI sequences B0, B500, and B1000 were acquired, as was an ADC map to identify the ischemic core. FLAIR and T2 were also used as surrogate markers of the time elapsed from symptom onset. A T1 contrastenhanced MRA of the supra-aortic trunks and intra-cranial vessels was then obtained to screen for large-vessel occlusion. In cases of MR imaging contraindications, an unenhanced head CT, CTA, and CTP were performed. All MR and CT images were reviewed by 2 independent senior neuroradiologists who did not participate in the initial therapeutic management.

\section{Devices}

Stent retrievers were used as a first-line device. In 138 cases, the Solitaire FR revascularization device (Covidien, Irvine, California) was used. This device is a stent-based thrombectomy system with a closed-cell design and a longitudinal split section. Alternative stent retriever devices were used in 6 cases: the Revive ${ }^{20}$ (Codman \& Shurtleff, Raynham, Massachusetts) (4 cases) and the Trevo ${ }^{21,22}$ (Stryker, Kalamazoo, Michigan) (2 cases).

\section{Mechanical Thrombectomy Protocol}

All procedures were performed via a femoral artery approach and with the patients under general anesthesia. For the anterior circu- lation, an 8F or 9F Merci balloon-guided catheter (BGC; Concentric Medical) was inserted through a sheath. For the posterior circulation, a 6F Envoy guiding catheter (Codman \& Shurtleff) was placed through a sheath into the dominant, or navigable, vertebral artery. A 0.021-inch internal diameter microcatheter (Prowler Select Plus [Codman \& Shurtleff] or Vasco 21 [Balt, Montmorency, France]) was navigated distal to the point of occlusion over a 0.014-inch steerable microwire. A microcatheter angiographic run was then carried out to define the vascular bed distal to the thrombus. The stent retriever was then introduced through the microcatheter and the device deployed across the occluding thrombus. The microcatheter distal marker was positioned at the exact level of the proximal marker of the stent retriever. After deployment, the stent retriever was maintained in place for approximately 3-7 minutes to allow full device expansion. The stent retriever was fully deployed in all cases. An angiographic run was performed to evaluate the degree of temporary recanalization. The fully deployed stent retriever and the delivery microcatheter were then gently pulled back together and recovered through the guiding catheter. During the retrieval, the BGC was inflated to interrupt anterograde flow. Manual aspiration with a $50-\mathrm{mL}$ syringe was performed through the hemostatic valve during the retrieval to reverse the flow and to aspirate clot debris possibly lost in the guide catheter lumen. The integrity of the stent was consistently checked following each pass.

Successful recanalization was defined as TICI $2 \mathrm{~b}$ or $3 .{ }^{23}$ If the vessel was not reopened to at least TICI $2 b$ with a maximum of 5 passes, then the treatment was considered failed. All TICI scores were independently reviewed by a second neuroradiologist and adjudicated in cases of disagreement. Neither IV heparin nor intra-arterial fibrinolytics were administered at any time during the procedure, even if the recanalization attempt was unsuccessful. Groin punctures were routinely closed with an Angio-Seal (St. Jude Medical, Minnetonka, Minnesota).

Should intracranial stenosis occur, complementary standalone angioplasty was preferred with use of a Gateway balloon (Stryker), or an UltraFlow balloon (Covidien) $2.5 \mathrm{~mm}$. Inflation was cautiously done up to $6 \mathrm{~atm}$. Following an angiographic control, stent placement was considered only if immediate restenosis of the target artery associated with immediate reocclusion occurred. In these cases, a Wingspan stent (Boston Scientific, Natick, Massachusetts) was chosen and sized according to the target vessel size. Antiplatelet management consisted of 500 $\mathrm{mg}$ of aspirin IV during the procedure, and double antiplatelet was discussed after the 24-hour CT control in view of any serious hemorrhagic complications.

\section{Adverse Event Definition}

The types of hemorrhagic complications were defined according to the European Co-operative Acute Stroke Study-II (ECASS II). ${ }^{24}$ Symptomatic ICH was defined as parenchymatous hemorrhage (PH) 1 or $\mathrm{PH} 2$ hematomas and a $\geq 4$-point NIHSS score decline or death within the periprocedural period.

Embolic complications were defined as an angiographic occlusion in a previously unaffected vascular territory observed on the angiogram after clot removal and associated with new 
ischemic changes on 24-hour postprocedural CT or MR imaging.

All 24-hour CT/MR imaging scans were reviewed by a second neuroradiologist and adjudicated in cases of disagreement.

\section{Postoperative Management}

A CT scan was performed at the end of the procedure to screen for immediate hemorrhagic transformation. Airway support was immediately discontinued after the procedure to allow prompt neurologic re-evaluation. Mechanical ventilation was maintained in cases of posterior circulation stroke. No antiplatelet or heparin was administered during the first 24 hours. An additional CT or MR imaging examination was performed 24 hours after the procedure. If no hemorrhage was present, aspirin $160 \mathrm{mg} /$ day was administered. The length of stay was between 2 days and 1 month. The NIHSS score was then reported at discharge by a stroke practitioner.

\section{Statistical Analysis}

Patient characteristics were presented by use of median and range for continuous variables and frequencies and proportions for categoric variables. Statistical significance between recanalization failure and success and between complications and no complications was assessed by the Pearson $\chi^{2}$ test for categoric variables and the Mann-Whitney $U$ test or the Student $t$ test for continuous variables. Baseline NIHSS score was entered as a continuous variable. The significance of adding or removing a variable from the logistic model was determined by the maximum likelihood ratio test. ORs and their 95\% CIs were calculated. The goodness-of-fit of the models was assessed by use of the Hosmer and Lemeshow $\chi^{2}$ test. A level of $P<.05$ was considered statistically significant. Statistical analysis was performed by use of SAS software, version 9 (SAS Institute, Cary, North Carolina).

\section{RESULTS}

\section{Population}

From August 2009 to November 2011, a total of 144 consecutive patients (70 women and 74 men) aged 26-91 years (median age, 70 years; mean age, 67 years) were treated. Mean baseline NIHSS score was 16.6 (median, 18; range, 3-36).

\section{Acute MR Imaging}

MR imaging was performed in 142 of 144 patients. Two patients had contraindications to MR imaging and were assessed by CT, CTA, and CTP. The mean DWI-derived ASPECT score was 6.3 (range, 3-10). In 109 patients (75.7\%), the occlusion involved the anterior territory: MCA in 60 patients, ICA in 10 patients, ICA termination in 23 patients, and tandem occlusion in 16 patients. Acute occlusion of the basilar artery (BA) was noted in 35 patients $(24.3 \%)$.

\section{Protocol Deviation}

Protocol deviations were observed in 18 cases (12.5\%). Seven patients with an NIHSS score $<8$ were included: 3 cases of a symptomatic BA occlusion and 4 cases of pertinent motor deficit in the anterior circulation. Eleven patients were treated despite an ASPECT score $<5$. Among these patients ( 7 patients from the combined group, 4 from the stand-alone thrombectomy group),
Relationship between perioperative mortality, failure, complications (embolic and hemorrhagic), and intracranial stenosis

\begin{tabular}{lccc}
\hline \multicolumn{1}{c}{ Population } & $\begin{array}{c}\text { Perioperative } \\
\text { Mortality (\%) }\end{array}$ & $\begin{array}{c}\text { P Value } \\
\text { at Discharge }\end{array}$ & $\begin{array}{c}\text { Mean NIHSS } \\
\text { at cohort }\end{array}$ \\
\hline Overall con & 18.4 & & 7.9 \\
Failure & 45 & .003 & 14.6 \\
Embolic complication & 38.9 & .0176 & 10.7 \\
Hemorrhagic complication & 45.5 & .0236 & 14.2 \\
Intracranial stenosis & 57.1 & .0176 & 16.3 \\
\hline
\end{tabular}

3 (21.4\%) underwent hemorrhagic transformation, from which 2 (18.2\%) were symptomatic.

\section{Failure Rate}

We noted 6 cases of TICI $2 \mathrm{a}(4.9 \%), 1$ case of TICI $1(0.7 \%)$, and 13 cases of TICI 0 (9\%), which corresponds to an observed total failure rate of $13.9 \%$. In our study, the mortality rate in failed thrombectomy cases $(45 \%)$ was significantly higher than in the overall cohort $(18.4 \% ; P=.003)$. The overall rate of successful recanalization was $86.1 \%$ (TICI 3: 69.4\%; TICI 2b: $16.7 \%$ ).

\section{Embolic and Hemorrhagic Complications}

Embolic complications were observed in 18 patients (12.5\%): 8 embolic complications (44.4\%) were observed in the stand-alone thrombectomy group and $10(55.6 \%)$ in the combined group $(P=.354)$ (Table and On-line Table). When the clot was removed from the anterior circulation (MCA or ICA), we reported distal emboli within the anterior cerebral artery territories ( 6 cases) (Fig 2), distal M3-MCA (3 cases), and posterior cerebral arteries (3 cases). When the clot was removed from the BA, embolic complications were observed within the posterior inferior cerebellar artery ( 3 cases), the posterior cerebral arteries ( 2 cases), and the superior cerebellar artery ( 2 cases). Thirteen patients $(72.2 \%)$ had clinical worsening including 7 deaths $(38.9 \%)$, whereas the overall rate of periprocedural mortality was $18.4 \%(P=.0176)$. Overall hemorrhagic complications were noted in 30 cases $(20.8 \%)$ : 9 $(18 \%)$ in the stand-alone thrombectomy group and $21(22.3 \%)$ in the combined group $(P=.541)$. We identified 4 subarachnoid hemorrhages, 6 hemorrhagic infarcts category 1 (HI1), 3 HI2, 9 $\mathrm{PH} 1$, and $8 \mathrm{PH} 2$ (Fig 1) according to the ECASS II classification. ${ }^{24}$ Eleven patients (7.6\%) had symptomatic ICH: 6 patients from the combined group and 5 patients from the stand-alone thrombectomy group. All ICHs were observed in the initial stroke territory. In our study, the mortality rate in patients with symptomatic ICH was $45.5 \%(P=.0236)$.

\section{Other Periprocedural Complications}

Technical complications were reported in 5 patients. In 1 patient, an in-stent thrombosis occurred 6 hours after stent deployment for an MCA dissection secondary to MCA stenosis angioplasty, resulting in clinical worsening. An iatrogenic extracranial ICA dissection resulted in an ICA occlusion, with the patient remaining asymptomatic because of good collaterals from the circle of Willis. An arterial perforation was reported in 1 patient, who died subsequently. This perforation was not caused by the thrombectomy device but, instead, by an MCA complementary angioplasty. Device fracture and spontaneous release of the stent retriever were also seen in 2 patients. In both cases, the device was left in place 

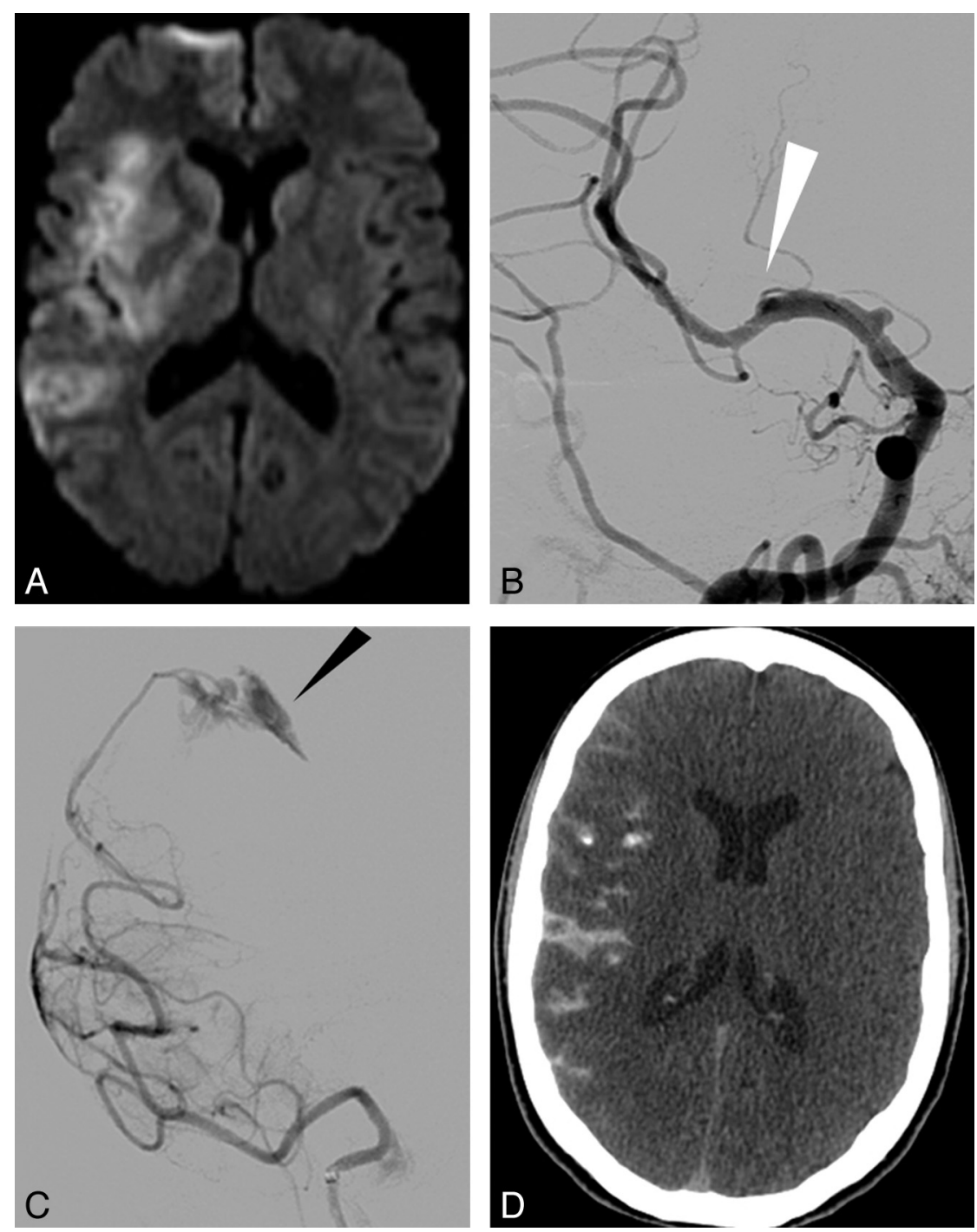

FIG 1. Rupture of a cortical pial vessel following selective injection via microcatheter. $A$, Initial DWI showing right MCA infarct (DWI ASPECT score, 5) without intracranial hemorrhage. $B$, Occlusion of right MCA (M2) (white arrow) seen on frontal angiogram. C, Selective injection via the microcatheter beyond the clot leading to rupture of a cortical pial vessel (black arrow). D, Immediate postoperative CT showing contrast within the subarachnoid space.

and the patient received long-term antiplatelet therapy with a good neurologic outcome at discharge.

No death or transfusion was related to groin puncture hematoma.

The rate of overall complications (including embolic complications, symptomatic ICH, and periprocedural complications) was $18.1 \%$. The cumulative rate of failure, complication, and/or death during the perioperative period was $39.6 \%$. Sixteen patients (11.8\%) died without procedural complications and $9(6.6 \%)$ secondary to a procedure-related event.

\section{Intracranial Stenosis}

Eight cases (5.5\%) of intracranial stenosis were observed, including 3 stenoses of the BA and 5 of the MCA. The mean age of the intracranial stenosis patients was 74.4 years (age range, 55-86 years). In the elderly population ( $>70$ years old), the overall mortality rate was $24.2 \%$, and the mortality rate related to intracranial stenosis in this subgroup was $18.8 \%$. Unsuccessful recanalization was observed in $7(87.5 \%)$ of 8 cases. Angioplasties were performed in 3 of 7 cases of intracranial stenosis and provided a TICI $2 b-3$ recanalization in 1 case. Additional deployment of an intracranial stent was performed in 1 case of intracranial stenosis with an acute in-stent thrombosis 3 hours postprocedure. Periprocedural complications were reported in $28.6 \%$ of cases and were related to complications of angioplasty (1 dissection and 1 arterial rupture). The mortality rate in cases of intracranial stenosis was $50 \%$.

\section{Clinical Evaluation at Discharge}

A total of 136 patients were assessed by a stroke practitioner at discharge. Mean NIHSS score at discharge was 7.9 (range, $0-25$ ) in the overall cohort vs 10.7 in cases of embolic complications and 14.2 for hemorrhagic complications. In cases of thrombectomy failure, the mean NIHSS score was 14.6; in patients with intracranial stenosis, it was 16.3. At discharge, 25 deaths (18.4\%) were reported (Table).

\section{DISCUSSION}

Recent studies (IMS III, ${ }^{25}$ SYNTHESIS, ${ }^{26}$ MR RESCUE trial ${ }^{27}$ ) did not observe superiority of endovascular approaches compared with standard intravenous rtPA treatment for large-vessel occlusion. However, these studies were conducted with first-generation devices, with almost no stent retriever. As recent studies ${ }^{22,28,29}$ have reported, there is a significant superiority of stent retriever devices compared with "non-stent retriever" devices from a clinical and technical point of view. Hence, the results from these trials have not changed our current treatment protocol and practice, and we are pending the results of further trials such as THRACE (Trial and Cost Effectiveness Evaluation of Intra-arterial Thrombectomy in Acute Ischemic Stroke), SWIFT PRIME (Solitaire FR as Primary Treatment for Acute Ischemic Stroke), MRCLEAN (Endovascular treatment for acute ischemic stroke in the Netherlands), and PISTE (Pragmatic Ischaemic Stroke Thrombectomy Evaluation).

\section{Recanalization Failure}

The recanalization failure rate observed (13.9\%) was roughly similar to the recent stent retriever literature (mean rate, $10.3 \%$; range, $0 \%-33.3 \%){ }^{15,20,22,28-31}$ It is not clear to date if IV and intra-arterial therapies are synergistic in achieving a higher recanalization rate.

In our study, IV thrombolytics were used in combination with mechanical thrombectomy in $65.3 \%$ of patients. In this group, the 

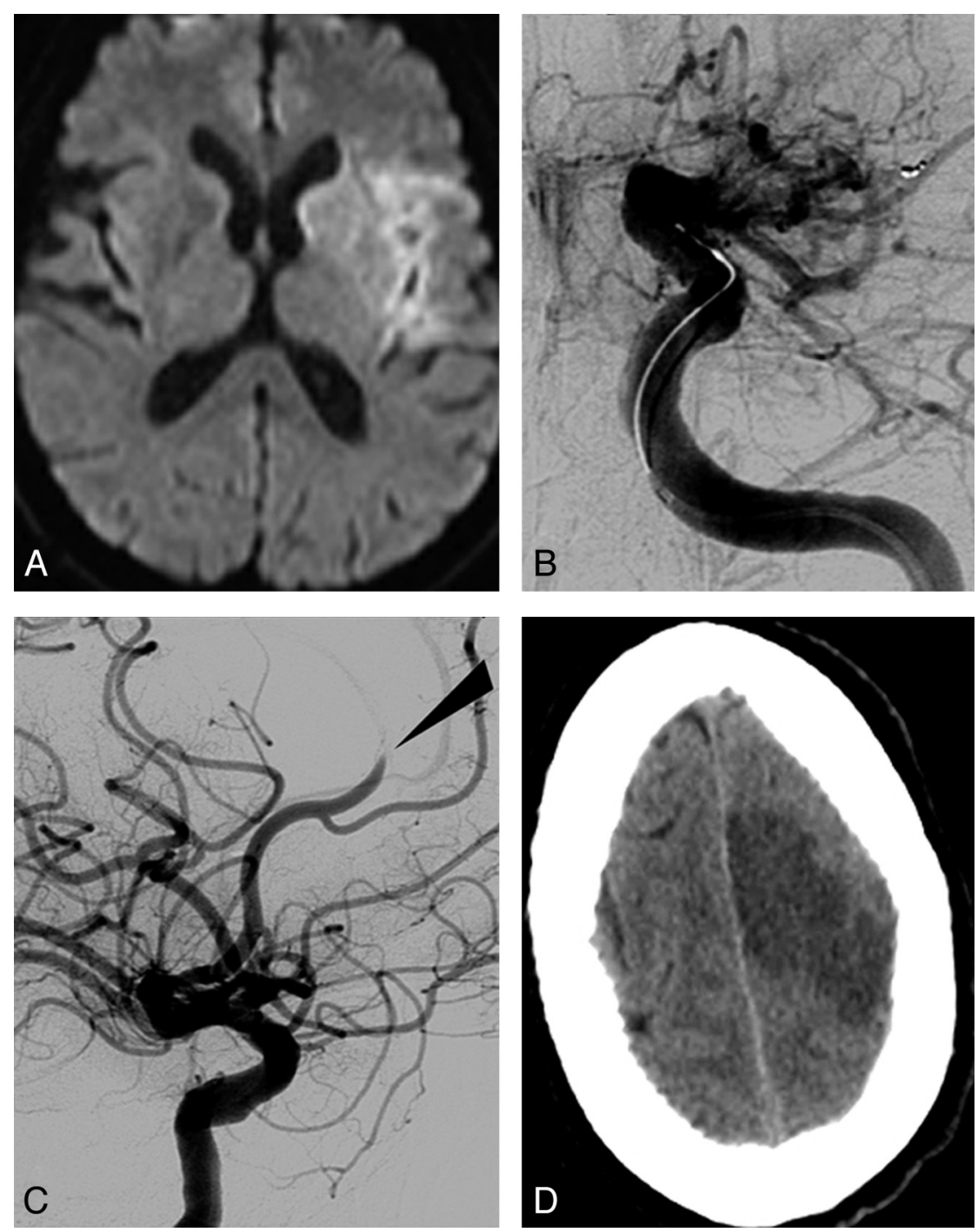

FIG 2. A thromboembolic occlusion of the left anterior cerebral artery after clot removal from the ipsilateral MCA. A, Initial DWI showing ischemic lesion in the left MCA territory (DWI ASPECT score, 7). B, Left ICA Townes projection angiogram showing terminal occlusion. $C$, Lateral projection angiogram after removal of the device allowing flow restoration within the MCA but embolic migration within the left anterior cerebral artery (arrow). D, New ischemic lesion seen on CT after the procedure within the left anterior cerebral artery territory.

fully efficient during mechanical treatment. It is interesting to note that Dávalos et $\mathrm{al}^{13}$ reported a significantly better 3 -month outcome $(\mathrm{mRS} \leq 2)$ in patients treated by means of combined therapy compared with the stand-alone group, suggesting that IV therapy could play a role at a "microcirculatory level" on brain reperfusion, not visible on final DSA control.

The failure rate did not reach any statistical difference between the anterior circulation $(12.3 \%)$ and the posterior circulation $(17.1 \%)(P=.535)$, despite the obvious anatomic difference between these 2 territories and the lack of a BGC in the posterior circulation.

\section{Embolic Complications}

In our study, embolic complications occurred in 18 cases $(12.5 \%)$ and seemed to be a major procedural issue of the technique despite such complications being rarely reported in the recent literature. For example, Castaño et $\mathrm{al}^{11}$ and Roth et $\mathrm{al}^{18}$ did not observe any embolic complications in their first experiences. Recently, Dorn et $\mathrm{al}^{14}$ and Nogueira et al ${ }^{22}$ observed a rate of embolic complications of $3.7 \%$ and $7 \%$, respectively. In an experimental model of cerebrovascular occlusion, authors compared 5 thrombectomy devices $^{32}$ and concluded that the risk for embolic showers was influenced by the mechanism of action of the thrombectomy device. There was a significant increase in the number of large clot fragments with the Penumbra system (Penumbra, Alameda, California) compared with the Merci retriever. The Solitaire FR device

recanalization failure rate was $14.9 \%$ vs $12 \%$ in patients treated by stand-alone thrombectomy $(P=.615)$. This result suggests that IV therapy did not significantly influence the recanalization rate in acute thrombectomy cases by using a stent retriever. In a similar fashion, Dávalos et $\mathrm{al}^{13}$ did not report any statistically significant difference between patients treated with combined therapy compared with the stand-alone thrombectomy group (failure rate, $14 \%$ vs $19 \%$ ). On the contrary, Dorn et al ${ }^{14}$ observed that the recanalization rate was higher when IV fibrinolysis was administered before mechanical thrombectomy. A reliable comparison between combined therapy and stand-alone thrombectomy is difficult to establish without a randomized trial. Actually, patients treated by stand-alone thrombectomy are selected following IV therapy exclusion criteria, resulting in a systematic selection bias. It is important to notice that the timing between these 2 therapies was not consistent and could reach several hours. In these conditions, we can hypothesize that IV therapy was not systematically produced larger fragments than the Waveguide system (OmniSonics Medical Technologies, Wilmington, Massachusetts). In our study, the mortality rate among patients who had embolic complications $(38.9 \%)$ was higher than in the overall cohort $(18.4 \%)(P=.0176)$. Eleven embolic events in 109 procedures were reported in the anterior circulation $(10.1 \%)$ and 7 cases (20\%) among 35 procedures in the posterior circulation, with no statistical difference but a slight tendency to a higher occurrence in the posterior circulation territory $(P=.144)$, as was already suggested. ${ }^{12}$ In the anterior circulation, Dávalos et al reported a significant role for BGCs in decreasing the incidence of collateral infarction in the anterior circulation. In the posterior circulation, in most circumstances, the bilateral supply from the contralateral vertebral artery prevents a complete blood flow reversal. The difference in embolic complication rates between the anterior and posterior circulation may also be explained by a less efficient aspiration in BA occlusion during clot removal. 
No statistical differences in embolic complications were observed between the combined group and the stand-alone thrombectomy group $(P=.354)$. Adjunctive fibrinolysis was not a significant protective factor as we may have initially expected.

\section{Hemorrhagic Complications}

Overall hemorrhagic complications occurred in $20.8 \%$ of patients, and symptomatic ICH was reported in $7.6 \%$ of patients. This rate $(7.6 \%)$ was roughly similar to the recent stent retriever literature (mean rate, 8.7\%; range, 0\%-20\%). ${ }^{15,20,22,28-31}$ The rate was only $4 \%$ in the Solitaire retrospective multicenter study. ${ }^{13}$ In our study, the mortality rate in the symptomatic ICH subgroup was significantly higher than in the overall cohort (45.5\% vs $18.4 \% ; P=.0236)$. It is not surprising that age was associated with an increased risk for ICH. Mean age in the hemorrhagic group was 72 years (median age, 74.5 years; age range, $40-89$ years) vs a mean age of 66 years (median age, 68.5 years; age range, $26-90$ years) in patients without hemorrhagic complication $(P=.043)$. As observed in previous IV fibrinolysis studies, ${ }^{33-36}$ age seemed to be an independent predictor of ICH in our series. ICH was not significantly different between the standalone and combined thrombectomy groups $(P=.615)$ suggesting that IV lytics did not promote an increased rate of ICH. Time lapsed from symptom onset to successful recanalization was also not related to hemorrhagic transformation, possibly because patient selection was carried out by use of DWI MR imaging, hence excluding large brain infarction.

Initial infarct size and volume measured on DWI MR imaging were reported as independent factors associated with hemorrhagic transformation in acute ischemic stroke. ${ }^{10,36,37}$ Actually, in our study, among 11 patients who had a DWI ASPECT score $<5,3$ $(27.3 \%)$ had hemorrhagic complications, and 2 (18.2\%) were symptomatic vs only $7.6 \%$ in the overall series $(P=.651)$.

\section{Intracranial Stenosis}

In previous stent retriever thrombectomy studies, ${ }^{11-17}$ intracranial stenoses were not discussed. In our series, intracranial stenoses were observed in 5.5\% of patients and were linked with a high mortality rate $(50 \%)$. The death rate in this subgroup of patients was significantly higher than in the overall cohort $(18.4 \% ; P=$ $.0176)$ and may be related to a substantial number of failed recanalizations $(87.5 \%)$ and periprocedural complications (28.6\%). Multiple procedural clot removal attempts in such cases may further disrupt an instable plaque and irreversibly damage the vessel wall. Nowadays, MR imaging or CTA could easily detect intracranial stenosis when arteries remain permeable ${ }^{38-40}$ but are still insufficient in diagnosing an underlying intracranial stenosis when the vessel is occluded.

\section{Study Limitations}

We acknowledge that our results need to be cautiously interpreted because they were derived from a single-center clinical experience. As with all continuous, prospectively collected clinical data, protocol deviations were observed (12.5\%) but were reported in the study (DWI-derived ASPECT score and NIHSS inclusion). Also, interpretation of the angiographic and MR imaging results, though systematically carried out by 2 experienced neuroradiologists, were not analyzed by an independent core laboratory.

\section{CONCLUSIONS}

In this single-center prospective stent retriever study, recanalization failure was reported in $13.9 \%$ of patients. Embolic and symptomatic hemorrhagic complications were observed in $12.5 \%$ and $7.6 \%$, respectively. The cumulative rate of failure, complication, and/or death was $39.6 \%$. Mortality rate was $38.9 \%$ in cases of embolic events, $45.5 \%$ in cases of symptomatic ICH, and $57.1 \%$ in cases of intracranial stenosis. Age was an independent predictor of hemorrhagic complications. Concomitant fibrinolytic therapy did not influence the rates of recanalization, collateral embolic complications, or ICH occurrence.

Disclosures: Alain Bonafé-UNRELATED: Consultancy: Covidien. Vincent CostalatUNRELATED: Consultancy: Covidien; Payment for Lectures (including service on speaker bureaus): ev3, J\&J (Codman); Payment for Development of Educational Presentations: Covidien; Travel/Accommodations/Meeting Expenses Unrelated to Activities Listed: Covidien, Stryker, Codman.

\section{REFERENCES}

1. Molina CA, Montaner J, Arenillas JF, et al. Differential pattern of tissue plasminogen activator-induced proximal middle cerebral artery recanalization among stroke subtypes. Stroke 2004;35:486-90

2. Tandberg Askevold E, Naess H, Thomassen L. Predictors for recanalization after intravenous thrombolysis in acute ischemic stroke. $J$ Stroke Cerebrovasc Dis 2007;16:21-24

3. Kimura K, Iguchi Y, Yamashita S, et al. Atrial fibrillation as an independent predictor for no early recanalization after IV-t-PA in acute ischemic stroke. J Neurol Sci 2008;267:57-61

4. Kimura K, Iguchi Y, Shibazaki K, et al. Early stroke treatment with IV t-PA associated with early recanalization. J Neurol Sci 2010; 295:53-57

5. Linfante I, Llinas RH, Selim M, et al. Clinical and vascular outcome in internal carotid artery versus middle cerebral artery occlusions after intravenous tissue plasminogen activator. Stroke 2002;33:2066-71

6. Rha JH, Saver JL. The impact of recanalization on ischemic stroke outcome: a meta-analysis. Stroke 2007;38:967-73

7. Zangerle A, Kiechl S, Spiegel M, et al. Recanalization after thrombolysis in stroke patients: predictors and prognostic implications. Neurology 2007;68:39-44

8. Kimura K, Sakamoto Y, Aoki J, et al. Clinical and MRI predictors of no early recanalization within 1 hour after tissue-type plasminogen activator administration. Stroke 2011;42:3150-55

9. Tanne D, Kasner SE, Demchuk AM, et al. Markers of increased risk of intracerebral hemorrhage after intravenous recombinant tissue plasminogen activator therapy for acute ischemic stroke in clinical practice: the Multicenter rt-PA Stroke Survey. Circulation 2002;105:1679-85

10. Singer OC, Humpich MC, Fiehler J, et al. Risk for symptomatic intracerebral hemorrhage after thrombolysis assessed by diffusionweighted magnetic resonance imaging. Ann Neurol 2008;63:52-60

11. Castaño C, Dorado L, Guerrero C, et al. Mechanical thrombectomy with the Solitaire AB device in large artery occlusions of the anterior circulation: a pilot study. Stroke 2010;41:1836-40

12. Costalat V, Machi P, Lobotesis K, et al. Rescue, combined, and standalone thrombectomy in the management of large vessel occlusion stroke using the Solitaire device: a prospective 50-patient singlecenter study: timing, safety, and efficacy. Stroke 2011;42:1929-35

13. Dávalos A, Pereira VM, Chapot R, et al. Retrospective multicenter study of Solitaire FR for revascularization in the treatment of acute ischemic stroke. Stroke 2012;43:2699-705

14. Dorn F, Stehle S, Lockau H, et al. Endovascular treatment of acute 
intracerebral artery occlusions with the Solitaire stent: single-centre experience with 108 recanalization procedures. Cerebrovasc Dis 2012;34:70-77

15. Koh JS, Lee SJ, Ryu CW, et al. Safety and efficacy of mechanical thrombectomy with Solitaire stent retrieval for acute ischemic stroke: a systematic review. Neurointervention 2012;7:1-9

16. Machi P, Costalat V, Lobotesis K, et al. Solitaire FR thrombectomy system: immediate results in $\mathbf{5 6}$ consecutive acute ischemic stroke patients. J Neurointerv Surg 2012;4:62-66

17. Stampfl S, Hartmann M, Ringleb PA, et al. Stent placement for flow restoration in acute ischemic stroke: a single-center experience with the Solitaire stent system. AJNR Am J Neuroradiol 2011;32: 1245-48

18. Roth C, Papanagiotou P, Behnke S, et al. Stent-assisted mechanical recanalization for treatment of acute intracerebral artery occlusions. Stroke 2010;41:2559-67

19. Nezu T, Koga M, Nakagawara J, et al. Early ischemic change on CT versus diffusion-weighted imaging for patients with stroke receiving intravenous recombinant tissue-type plasminogen activator therapy: Stroke Acute Management with Urgent Risk-factor Assessment and Improvement (SAMURAI) rt-PA registry. Stroke 2011;42:2196-200

20. Rohde S, Haehnel S, Herweh C, et al. Mechanical thrombectomy in acute embolic stroke: preliminary results with the Revive device. Stroke 2011;42:2954-56

21. San Román L, Obach V, Blasco J, et al. Single-center experience of cerebral artery thrombectomy using the TREVO device in 60 patients with acute ischemic stroke. Stroke 2012;43:1657-59

22. Nogueira RG, Lutsep HL, Gupta R, et al. Trevo versus Merci retrievers for thrombectomy revascularisation of large vessel occlusions in acute ischaemic stroke (TREVO 2): a randomised trial. Lancet 2012;380:1231-40

23. Higashida RT, Furlan AJ, Roberts $\mathrm{H}$, et al. Trial design and reporting standards for intra-arterial cerebral thrombolysis for acute ischemic stroke. Stroke 2003;34:e109-37

24. Hacke W, Kaste M, Fieschi C, et al. Randomised double-blind placebo-controlled trial of thrombolytic therapy with intravenous alteplase in acute ischaemic stroke (ECASS II). Second EuropeanAustralasian Acute Stroke Study Investigators. Lancet 1998;352: 1245-51

25. Broderick JP, Palesch YY, Demchuk AM, et al. Endovascular therapy after intravenous t-PA versus t-PA alone for stroke. $N$ Engl J Med 2013;368:893-903

26. Ciccone A, Valvassori L, Nichelatti M, et al. Endovascular treatment for acute ischemic stroke. N Engl J Med 2013;368:904-13

27. Kidwell CS, Jahan R, Alger JR, et al. Design and rationale of the
Mechanical Retrieval and Recanalization of Stroke Clots Using Embolectomy (MR RESCUE) Trial. Int J Stroke 2014;9:110-16

28. Broussalis E, Trinka E, Hitzl W, et al. Comparison of stent-retriever devices versus the Merci retriever for endovascular treatment of acute stroke. AJNR Am J Neuroradiol 2013;34:366-72

29. Saver JL, Jahan R, Levy EI, et al. Solitaire flow restoration device versus the Merci retriever in patients with acute ischaemic stroke (SWIFT): a randomised, parallel-group, non-inferiority trial. Lancet 2012;380:1241-49

30. Ribo M, Molina CA, Jankowitz B, et al. Stentrievers versus other endovascular treatment methods for acute stroke: comparison of procedural results and their relationship to outcomes. J Neurointerv Surg 2013 May 28. [Epub ahead of print]

31. Mordasini P, Brekenfeld C, Byrne JV, et al. Technical feasibility and application of mechanical thrombectomy with the Solitaire FR revascularization device in acute basilar artery occlusion. AJNR Am J Neuroradiol 2013;34:159-63

32. Chueh JY, Wakhloo AK, Gounis MJ. Effectiveness of mechanical endovascular thrombectomy in a model system of cerebrovascular occlusion. AJNR Am J Neuroradiol 2012;33:1998-2003

33. Bluhmki E, Chamorro A, Davalos A, et al. Stroke treatment with alteplase given 3.0-4.5 $\mathrm{h}$ after onset of acute ischaemic stroke (ECASS III): additional outcomes and subgroup analysis of a randomised controlled trial. Lancet Neurol 2009;8:1095-102

34. Derex L, Nighoghossian N. Intracerebral haemorrhage after thrombolysis for acute ischaemic stroke: an update. J Neurol Neurosurg Psychiatry 2008;79:1093-99

35. Intracerebral hemorrhage after intravenous t-PA therapy for ischemic stroke. The NINDS t-PA Stroke Study Group. Stroke 1997;28:2109-18

36. Larrue V, von Kummer RR, Muller A, et al. Risk factors for severe hemorrhagic transformation in ischemic stroke patients treated with recombinant tissue plasminogen activator: a secondary analysis of the European-Australasian Acute Stroke Study (ECASS II). Stroke 2001;32:438-41

37. Sakamoto Y, Kimura K, Iguchi Y, et al. Hemorrhagic transformation in acute cerebellar infarction. Cerebrovasc Dis 2011;32:327-33

38. $\mathrm{Xu} \mathrm{WH,} \mathrm{Li} \mathrm{ML,} \mathrm{Gao} \mathrm{S,} \mathrm{et} \mathrm{al.} \mathrm{In} \mathrm{vivo} \mathrm{high-resolution} \mathrm{MR} \mathrm{imaging} \mathrm{of}$ symptomatic and asymptomatic middle cerebral artery atherosclerotic stenosis. Atherosclerosis 2010;212:507-11

39. Chung GH, Kwak HS, Hwang SB, et al. High resolution MR imaging in patients with symptomatic middle cerebral artery stenosis. Eur J Radiol 2012;81:4069-74

40. Marquering HA, Nederkoorn PJ, Smagge L, et al. Performance of semiautomatic assessment of carotid artery stenosis on CT angiography: clarification of differences with manual assessment. AJNR Am J Neuroradiol 2012;33:747-54 